\title{
Fatal neonatal persistent pulmonary hypertension caused by vegetations of infective endocarditis - case report and review of the literature
}

\author{
Andreea Luciana Avasiloaiei ${ }^{*}, 1,2$, Ecaterina Iftime ${ }^{2}$, Daniela Claudia Scripcaru ${ }^{3}$, Andrei \\ Petrariu $^{2}$, Gabriela Ildiko Zonda ${ }^{1,2}$, Maria Stamatinn², Luminița Păduraru ${ }^{1,2}$ \\ ${ }^{1}$ Department of Mother and Child Health, "Grigore T. Popa" University of Medicine and Pharmacy, lasi, \\ Romania; ${ }^{2}$ Neonatal Intensive Care Unit, Cuza-Voda Clinical Hospital of Obstetrics and Gynecology, \\ lasi; ${ }^{3}$ Pathology Department, Cuza-Voda Clinical Hospital of Obstetrics and Gynecology, lasi, Romania
}

\begin{abstract}
Neonatal infective endocarditis is a rare condition and usually pertains to a specific class of immunologically depressed preterm infants, with a long history of invasive procedures in the Neonatal Intensive Care Unit. We report the case of an aggressive and fatal neonatal infective endocarditis in a full-term infant, who developed massive endocardial vegetations on the tricuspid valve, leading to persistent pulmonary hypertension of the newborn, unresponsive to nitric oxide ventilation. Post-mortem cardiac cultures were positive with Serratia marcescens, an unusual germ for an early-onset infection, which was absent in blood cultures.
\end{abstract}

Keywords: neonatal infective endocarditis; persistent pulmonary hypertension of the newborn; endocardial vegetations; Serratia marcescens

\section{Introduction}

Neonatal infective endocarditis (NIE) is a more common occurrence in preterm infants, usually after a long hospitalization in the Neonatal Intensive Care Unit (NICU). The variable clinical features of NIE are often nonspecific, mimicking those of sepsis or congenital heart disease. When cardiac ultrasound cannot be performed, the diagnosis of NIE relies mainly on blood cultures, since diagnostic criteria for endocarditis are not very useful in the neonatal period [1]. We report a case of neonatal endocarditis, with an unusual germ, in a term neonate.

Received: June 2020; Accepted after review: August 2020; Published: August 2020.

${ }^{*}$ Corresponding author: Andreea Luciana Avasiloaiei, Neonatal Intensive Care Unit, Cuza-Voda Clinical Hospital of Obstetrics and Gynecology, Str. Cuza Vodă 34, 700038 lasi, Romania

Email: andreea avasiloaiei@ymail.com

\section{Case report}

A full-term female infant was referred to our regional level III NICU at 36 hours of life. The birth occurred by elective Caesarean section in a third level maternity hospital, at 39 weeks gestation, following an uneventful pregnancy, with no evidence of infection in the mother, with ruptured membranes two hours prior to surgery. The neonate had a birth weight of 2600 grams, showing no weight gain during the last three weeks of pregnancy, according to serial ultrasound examinations performed by the same obstetrician. The amniotic fluid was meconium-stained, but with no evidence of aspiration by the infant and it was not cultured, so there is no evidence for a possible chorioamnionitis. The umbilical cord and the placenta were normal at gross examination.

After birth, the infant developed progressive respiratory distress and was intubated after initial oxygen administrated by 
hood. Despite conventional mechanical ventilation, the postductal $\mathrm{SpO} 2$ progressively dropped to $40 \%$ and our center was called to approve her admission. She maintained a precarious status throughout the transport period and was admitted with severe hypoxemia, a preductal $\mathrm{SpO} 2$ of $65-70 \%$ and a postductal SpO2 of $45-55 \%$. She had severely altered general status, with pale-mottled skin, important respiratory distress, tachypnea, a III/6 heart murmur and severe hypotonia. The initial echocardiogram revealed important pulmonary hypertension (39-44 $\mathrm{mmHg}$ ), severe tricuspid regurgitation (velocity $293 \mathrm{~cm} / \mathrm{sec}$, gradient $34 \mathrm{mmHg}$ ) and large patent foramen ovalae with right-to-left shunting. The cranial ultrasound reveals marked cerebral edema. The initial blood work revealed severe thrombocytopenia $(15,000 / \mathrm{mmc})$ and high values of urea, creatinine, liver enzymes, markers of tissue hypoxia and inflammation. Blood cultures, both at the referring hospital and in our center were sterile.
The neonate required high frequency oscillatory ventilation with an initial $\mathrm{FiO} 2$ of 1 and later nitric oxide at $20 \mathrm{ppm}$, pentoxiphylline, sildenafil, surfactant, ampicillin for antibiotic prophylaxis, dobutamine for hemodynamic support, sodium bicarbonate for the correction of metabolic acidosis, insulin for stress hyperglycemia, with no significant improvement of the general status and of the saturations.

The second cardiac ultrasound, performed by a different examiner 12 hours after admission, revealed beyond the initial findings, a hyperechogenic and mobile structure linked to the leaflets of the tricuspid valve (Figure 1). The high level of suspicion for pulmonary thromboembolism mandated sampling of Ddimers, which showed extremely high values. The infant was started on intravenous heparin, with a slight and short-termed improvement of the general status and of the saturations (70$75 \%)$.

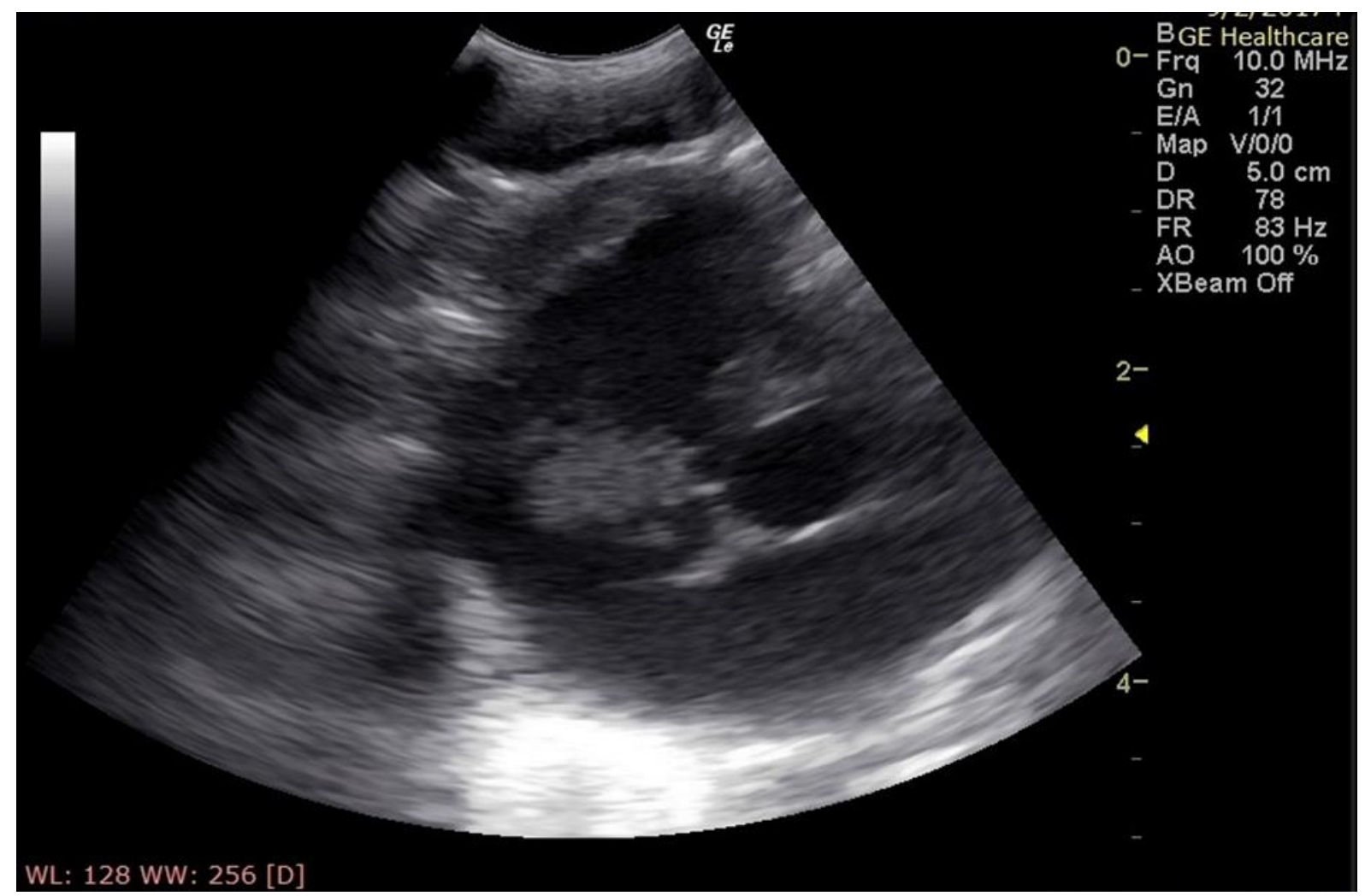

Fig. 1. Two-dimensional echocardiographic images of large hyperechogenic structures that loosely adhere to the leaflets of the tricuspid valve and protrude in the right atrium. 


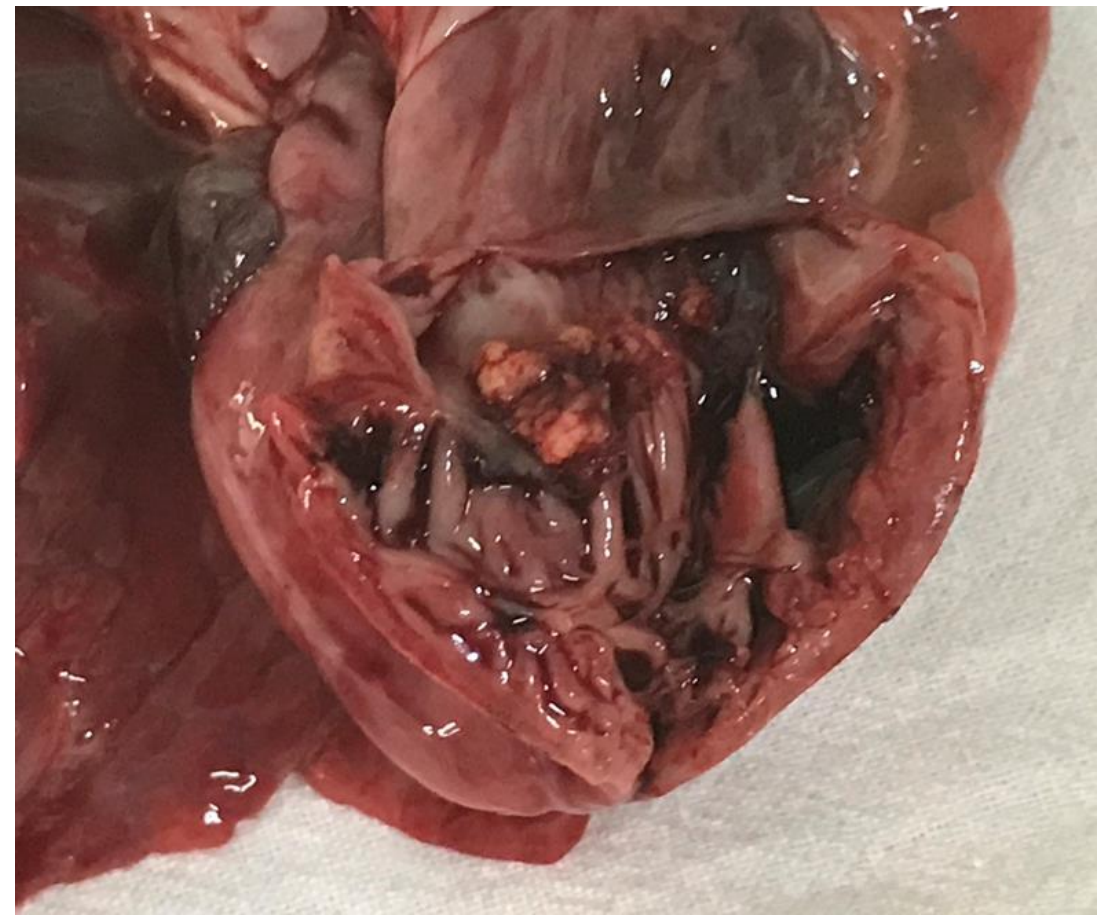

Fig. 2. Macroscopic examination of the right heart. Large endocardial vegetations are present on the atrial aspects of the tricuspid valve leaflets

At 60 hours after birth, the status severely and progressively deteriorated with profound metabolic acidosis, gasping breathing, lack of reactivity, undetectable saturations, both on preductal and postductal levels. The infant suffered multiple cardiopulmonary arrests, which needed complex resuscitation, but were ultimately fatal, at 63 hours of life.
The post-mortem examination revealed a normal sized heart, with massive endocardial vegetations on the leaflets of the tricuspid valve (Fig. 2) and multiple abscesses on both lungs. The microscopical examination of cardiac tissue showed that the vegetations were made up of fibrine, blood cells and bacteria (Fig. 3). The cultures in both sites were positive with Serratia marcescens.

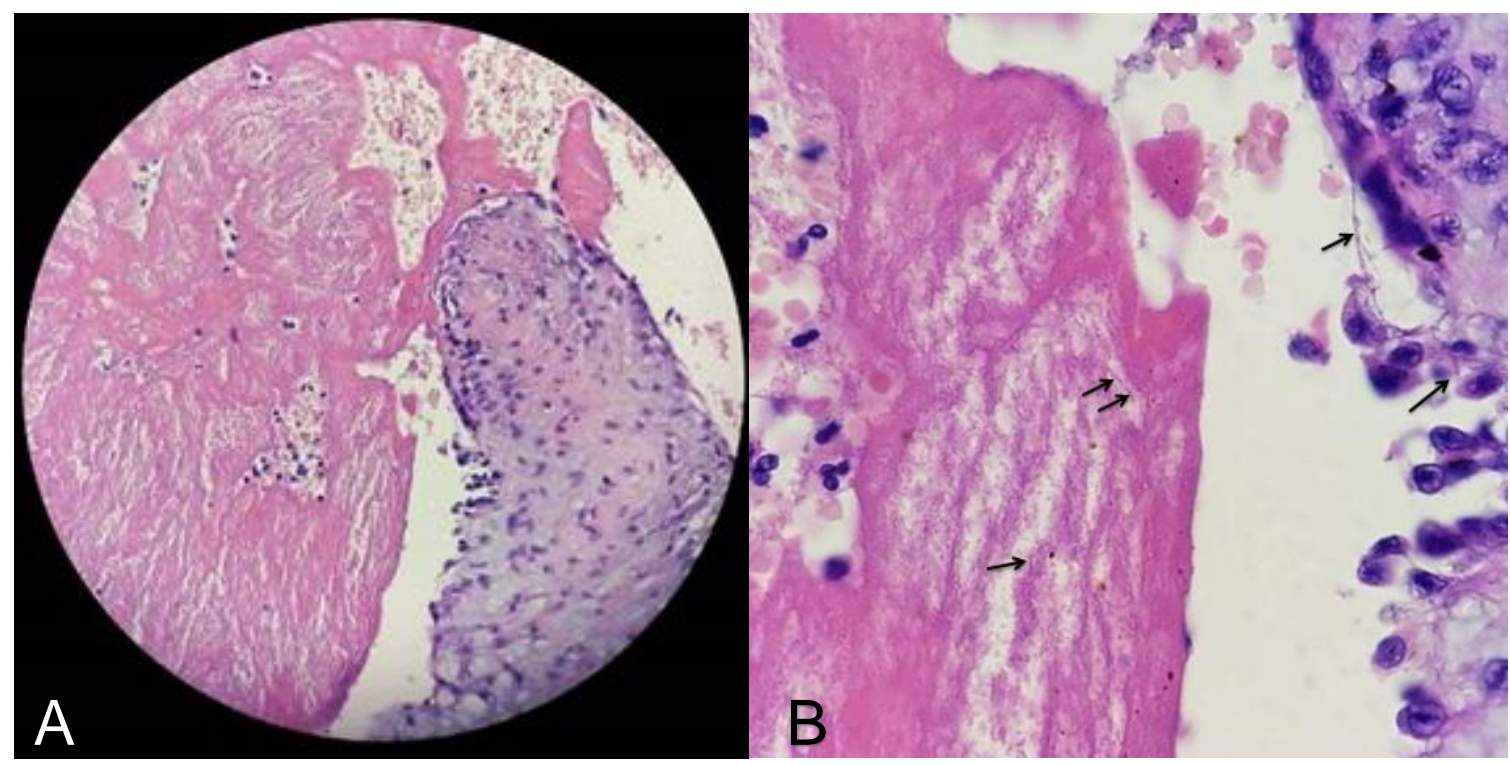

Fig. 3. Microscopic examination of the endocardial vegetations. A. Vegetation that adheres to the endocardium, represented by fibrine, erythrocytes, leukocytes and bacteria (hematoxylin-eosin, x100); B. Arrows point to existing bacilli in the vegetation and on the surface of the endocardium (hematoxylin-eosin, $x 400$ ) 


\section{Discussion}

NIE is a serious, but relatively rare infection that is associated with a high degree of morbidity and mortality. Its increased diagnosis in the past 30 years is mainly due to the increasing use of invasive techniques to manage neonates with multiple complex medical issues.

Most of the patients with NIE have an identifiable risk factor for the disease, such as congenital heart defects (CHD) [2-6], including patent ductus arteriosus (PDA) [7] and premature infants with central venous catheters (CVC), due to disruption of endocardium or valvular endothelial tissue, associated with mechanical trauma [2, 7-10].

In our case, NIE occurred in a welldocumented full-term pregnancy, with no adverse effects throughout and normal fetal ultrasounds. The only subtle suspicion could be considered the lack of fetal growth during the last three weeks of the pregnancy.

Staphylococci and streptococci are the most common pathogens during childhood [2, 11], but in neonates, Klebsiella pneumoniae and Enterobacter species may be added as etiologic agents [12, 13]. Serratia spp. was described as an isolated find in a 25-week gestation, 5-day old infant with PDA ligation and central venous line [7].

The term NIE is used for both bacterial and fungal endocarditis; the latter is caused mostly by Candida species, found mainly in fixed CVC and parenteral nutrition containing high glucose concentrations, but also Aspergillus, although to a lesser extent [7, 14].

The germ we identified in the infant's peripheral cultures and post-mortem cardiac cultures - Serratia marcescens - is unusual both in this location, and after such a short period of hospitalization time, in an infant who was not subjected to invasive vascular procedures. This is indeed the first case we are aware of Serratia marcescens infection, with such severe and early cardiac manifestations.

The clinical presentation of NIE is variable and nonspecific, leading to difficulties and delays in diagnosis unless a very high index of suspicion is observed. The symptoms are often indistinguishable from those of neonatal sepsis and Duke's modified criteria are often misleading [15], since blood cultures can be negative [1]. The most important orienting features are heart murmurs, hepatosplenomegaly, skin lesions, congestive cardiac failure, embolic events in the lungs or brain [16].

In our case, the initial respiratory distress in a term infant was probably considered a neonatal transient tachypnea which was treated with free flow oxygen delivered by hood. The respiratory condition quickly escalated, the infant showing signs of persistent pulmonary hypertension (PPHN), as ascertained by the differences in preductal and postductal saturations and confirmed by echocardiogram. PPHN was most probably determined by the large endocardial vegetations that obstructed blood flow at the level of the tricuspid valve.

It is unusual for the cardiac ultrasound to show endocardial vegetations in a two-day old neonate. These usually appear after 10-14 days, as reported by Daher et al. in their study of 16 infants [7]. In their study on 8 neonates with NIE, Opie et al. [17] reported a mean age of 10 days for echocardiographic diagnosis.

Also, in our case, vegetations appeared on a structurally normal heart, similarly to the case series by Mecrow and Ladurans [18]. The main difference resides in the exact location of endocardial vegetations, the tricuspid valve in our case, versus the junction of the superior vena cava and the right atrium, in all 12 cases they reported.

Vaideeswar et al. report a case of nonbacterial thrombotic endocarditis in a one-dayold late preterm infant delivered by Caesarean section for abnormal presentation and nonprogression of labor. The infant showed subsequent respiratory distress and died at 19 hours of life. The autopsy revealed vegetations on the atrial aspect of the tricuspid leaflets. The authors assume that the pathogenesis of the condition may be linked to hemodynamic disturbances due to perinatal stress [19]. In the case we present, chronic fetal distress may be expressed by the seemingly unnoticeable failure to thrive during the last three weeks of pregnancy, which manifested at birth as meconium-stained amniotic fluid.

In a historic series of 13 autopsy cases of non-infectious thrombotic endocarditis on structurally normal hearts, Morrow et al. remark a significant association between tricuspid 
vegetations, thrombocytopenia and persistent fetal circulation [20], which is highly similar to the situation we present. They postulate that turbulence at the tricuspid valve site due to right-to-left shunting, together with hypoxemia and activation of pro-aggregatory platelet substances, could account for the unique distribution of endocardial lesions in neonates, as well as for the short period of time in which they developed. Also, non-infective endocarditis may precede and predispose to infective endocarditis, by creating the location of pathogen attachment [1].

The prognosis of NIE is questionable most of the time. Early antibiotic therapy can impact favorably on the outcome. NIE with large, mobile vegetations is associated with a very high mortality rate due to a high risk of cerebral embolism and the diagnosis is often made at the post-mortem examination [10], as was our case. The evolution of our case was so acute, that we had no time to institute proper targeted antibiotic therapy.

\section{References}

1. Kumar N, Kumutha J, Ramamoorthy B, Gopaul S. Neonatal endocarditis: A unique encounter. Clin Pediatr 2002; 41:621-624.

2. Day MD, Gauvreau K, Shulman S, Newburger JW. Characteristics of children hospitalized with infective endocarditis. Circulation 2009; 119(6):865-870.

3. Marom D, Levy I, Gutwein O, et al. Healthcareassociated versus community-associated infective endocarditis in children. Pediatr Infect Dis J 2011; 30(7):585-588.

4. Saiman L, Prince A, Gersony WM. Pediatric infective endocarditis in the modern era. $J$ Pediatr 1993; 122:847-853.

5. Ware AL, Tani LY, Weng HY, et al. Resource utilization and outcomes of infective endocarditis in children. J Pediatr 2014; 165:807-812.

6. Baltimore RS, Gewitz M, Baddour, LM, et al. Infective Endocarditis in Childhood: 2015 Update. A scientific statement from the American Heart Association. Circulation 2015; 132:1487-1515.

7. Daher AH, Berkowitz FE. Infective endocarditis in neonates. Clin Pediatr (Phila) 1995; 34:198206.

8. Garcia-Teresa MA, Casado-Flores J, Delgado Domingues MA, et al. Infectious complications

\section{Conclusion}

This case report highlights an unusually rapid progression of neonatal infective endocarditis, as well as the unusual germ that caused it. Endocarditis should not be overlooked in a severely distressed neonate with no apparent risk factors, with evidence of inflammation and should mandate immediate diagnostic ultrasound.

\section{Consent}

Written informed consent was obtained from the patient for publication of this case report and accompanying images. A copy of the written consent is available for review by the Editor-in-Chief of this journal.

\section{Competing interests}

The authors declare that they have no competing interests. of percutaneous central venous catheterization in pediatric patients: a Spanish multicenter study. Intensive Care Med 2007; 33(3):466-476.

9. Rastogi A, Luken JA, Pildes RS, et al. Endocarditis in neonatal intensive care unit. Pediatr Cardiol 1993; 14:183-186.

10. Ferrieri $P$, Gewitz $M H$, Gerber MA, et al. Unique features of infective endocarditis in childhood. Circulation 2002; 105:2115-2127.

11. Johnson JA, Boyce TG, Cetta F, et al. Infective endocarditis in the pediatric patient: a 60-year single-institution review. Mayo Clin Proc 2012; 87(7):629-635.

12. Milazzo AS Jr, Li JS. Bacterial endocarditis in infants and children. Pediatr Infect Dis J 2001; 20:799-801.

13. Toganel R. Infective endocarditis in children New approach in antimicrobial prophylaxis. $J$ Interdisciplinary Med 2016; 1(1):9-11.

14. Azhar A. Successful management of fungal pericarditis and endocarditis in a neonate: $A$ case report. J Saudi Heart Assoc 2012; 24:195199.

15. Tissieres $P$, Gervaix $A$, Beghetti $M$, Jaeggi ET. Value and limitations of the von Reyn, Duke, and Modified Duke Criteria for the diagnosis of infective endocarditis in children. Pediatrics 2003; 112(6 Pt 1):e467. 
16. Nadroo AM, Al She Wear A; AI Sowailem AM, Al Jubair F. Infective endocarditis in a neonate. Annals Saudi Med 1997; 17(3):347-349.

17. Opie GF, Fraser SH, Drew JH, Drew S. Bacterial endocarditis in neonatal intensive care. $J$ Paediatr Child Health 1999; 35:545-548.

18. Mecrow IK, Ladusans EJ. Infective endocarditis in newborn infants with structurally normal hearts. Acta Paediatr 1994; 83:35-39.
19. Vaideeswar P, Sivaraman A, Deshpande JR. Non-bacterial thrombotic endocarditis in a neonate. Indian Pediatr 1998; 35:62-63.

20. Morrow WR, Haas JE, Benjamin DR. Nonbacterial endocardial thrombosis in neonates: Relationship to persistent fetal circulation. J Pediatr 1982; 100:117-122. 